\title{
BUCHBESPRECHUNGEN
}

\section{Andreas Zimmermann}

\section{Staatennachfolge in völkerrechtliche Verträge}

Zugleich ein Beitrag zu den Möglichkeiten und Grenzen völkerrechtlicher Kodifikation Springer Verlag, Berlin, 2000, 960 S., DM 298,--

Das Recht der Staatensukzession gilt unter den Teilgebieten des Völkerrechts seit langem vor allem deshalb als eines der schwierigsten, weil hier die Staatenpraxis sehr diffus und widersprüchlich, das Quellenmaterial, obwohl sehr umfangreich, so gut wie nicht erforscht und systematisiert, kurz: das Terrain für den Rechtsanwender sehr undankbar ist. Eigentlich ein Tummelplatz für Wissenschaftler, die eine akribische Sammel- und Sortierarbeit nicht scheuen, sollte man meinen. Und dennoch ist die hier anzuzeigende Heidelberger Habilitationsschrift von Zimmermann, soweit ersichtlich, die erste umfassende Monographie in deutscher Sprache, die es unternimmt, die Staatenpraxis zur Staatensukzession zusammenzustellen und zu analysieren. Den letzten ähnlich systematischen Anlauf unternahm der australische Völkerrechtler O'Connell in einem zweibändigen Werk vor über dreißig Jahren. Zimmermann beschränkt sich auf den Sukzessionsgegenstand der völkerrechtlichen Verträge, da zum einen hier das Praxismaterial besser als für andere Materien zugänglich ist und zum anderen ein Kodifikationsversuch der Vereinten Nationen in Gestalt der Wiener Konvention über das Recht der Staatennachfolge in Verträge von 1978 vorliegt, dessen Bewertung ihm ebenfalls ein Anliegen ist. Durch diese thematische Beschränkung ist die Darstellung auf 845 Textseiten noch gut handhabbar, der „Praxisbericht“ für die anderen Nachfolgematerien würde sicherlich denselben Umfang in Anspruch nehmen; hier bleibt also noch ein Betätigungsfeld für andere ,Jäger und Sammler“ des Völkerrechts.

Die Arbeit gliedert sich in vier Teile, deren erster (S. 9-130) den dogmatischen Vorklärungen zu Begriff und Formen der Staatennachfolge gewidmet ist. Der zweite Teil (S. 131219) betrachtet die historische Staatenpraxis bis zur Konvention 1978 sowie das Entstehen der Konvention selbst, während der dritte Teil (S. 221-818), das Herzstück der Arbeit, die Staatenpraxis unter dem Eindruck der Konvention, also nach 1978, darstellt. Der vierte Teil (S. 819-845) schließlich zieht die Summe und fragt nach dem Wert der Konvention für Kodifikation und Weiterentwicklung des Rechts der Staatensukzession. Der englische Text der Konvention sowie ein Sach- und Personenregister runden den Band im Anhang ab.

In seinem einleitenden Teil bestimmt Zimmermann den Tatbestand der Staatensukzession im Anschluß an die Definition in der Konvention (S. 15 f.) und stellt überblicksartig die verschiedenen Sukzessionsformen vor (S. 17-36). Den klassischen Formen Zession, Inkorporation, Fusion, Abspaltung und Dismembration stellt er das Konzept des newly inde- 
pendent state zur Seite, ein politisch-ideologisches Produkt der Dekolonisierungsperiode, welches die Konvention wie ein roter Faden durchzieht (S. 23 f.). Den Schwerpunkt der folgenden Abgrenzungsfragen bildet die in der Tat intrikate Unterscheidung von Sukzession und Identität (S. 46-130). Denn nur wenn wirklich ein Sukzessionstatbestand vorliegt, und nicht etwa eine Staatsidentität unter veränderten Rahmenbedingungen, kann das anfallende Praxismaterial für die Suche nach Regeln über die Rechtsfolgen der Staatensukzession verwendet werden. Zimmermann unterscheidet verschiedene Fallgruppen, in denen eine Identität in Frage stehen könnte, und wendet die bereits im Schrifttum entwickelten Abgrenzungskriterien auf bekannte Zweifelsfälle an. Mag er sich auch für die Unabhängigkeit der baltischen Staaten nicht festlegen (S. 57-60), so entscheidet er sich im Fall der Sowjetunion unter dem Eindruck der überwiegenden Staatenpraxis für eine Subjektsidentität der Russischen Föderation (S. 85-97) und im Fall Jugoslawiens nur sehr zögernd für Diskontinuität (S. 111 f.), was als Sukzessionskategorie zur Dismembration führt.

Der zweite, historische Teil skizziert nach Sukzessionsformen gegliedert die Staatenpraxis des 19. Jahrhunderts bis zum Ende des 2. Weltkriegs sowie der Zeit von 1945 bis 1978. Für die erste Zeitspanne ist die Praxis bereits im völkerrechtlichen Schrifttum, vor allem in den Arbeiten O'Connells aufbereitet worden. Zimmermann greift verständlicherweise darauf zurück und sieht zu Recht bereits vor 1945 einige Grundregeln etabliert, wie z.B. die beweglichen Vertragsgrenzen bei der Zession und die Kombination von Vertragserstreckung und Erlöschen bei der Inkorporation (S. 145); seine Annahme, bei der Dismembration habe sich eine Tendenz zur Vertragskontinuität gezeigt, ist allerdings durch das Praxismaterial kaum belegt. Die Zeit nach 1945 war maßgeblich durch die Praxis der Dekolonisierung geprägt. Zimmermann entnimmt ihr die „einheitliche Vorstellung“, daß völkerrechtliche Verträge des Kolonialherrn den newly independent state grundsätzlich nicht binden (clean-slate-Regel), es sei denn aufgrund einer einseitigen Erklärung oder eines konstitutiven Überleitungsabkommens (S. 164 f.). Für Zession und Inkorporation ergibt sich in diesem Zeitabschnitt eine Bestätigung der erwähnten Grundregeln, während sich für Abspaltungen das Erlöschen der Altverträge mit dem Recht des Neustaates zur einseitigen Sukzessionserklärung abzeichnete (S. 173). Eine Fortgeltung der Altverträge bei Fusionen läßt sich bis 1978 nicht nachweisen (S. 192 f.).

Nachdem so die völkergewohnheitsrechtliche Ausgangslage vor Abschluß der Wiener Konvention von 1978 umschrieben ist, geht es im anschließenden Kapitel um den Entstehungsprozeß der Konvention selbst (S. 194-219). Die verschiedenen Entwürfe und die Diskussion im Rahmen der UN-Völkerrechtskommission, auch auf der Staatenkonferenz 1977/78 werden knapp geschildert, ohne daß aber recht deutlich würde, warum die Konvention schließlich in weiten Teilen, etwa für die Vereinigung, den Zerfall von Staaten und für die Abspaltungsfälle, Regelungen aufstellt, die überhaupt nichts mit der vorangegangenen Staatenpraxis zu tun haben. So erscheint etwa die ausnahmslose Kontinuitätsregel in allen Fällen der Staatenvereinigung (Art. 31 der Konvention) plötzlich und für den Leser völlig unmotiviert im Fünften Bericht von Humphrey Waldock (S. 202 f.), ohne daß sich eine sachliche Anknüpfung dafür ausmachen ließe. Hier hätte man sich ein tieferes Nach- 
bohren in den Materialien von Kommission und Konferenz, vielleicht auch eine Art Motivforschung bei einzelnen ihrer Teilnehmer gewünscht.

Steht somit fest, daß die Regeln der Konvention über die Rechtsfolgen von Staatensukzessionen bei ihrer Abfassung zu einem großen Teil nicht der vorangegangenen Staatenpraxis entsprechen, so untersucht der nun folgende dritte Teil, inwieweit die Konventionsbestimmungen immerhin nach 1978 zur Richtschnur für die völkerrechtliche Staatenpraxis wurden. Auf diesem Weg könnte sich ergeben, daß die Konvention, wenn die - nicht konventionsgebundene - Praxis ihr weitgehend gefolgt ist, immerhin nachträglich zur ,gesicherten Kodifizierung geltenden Völkergewohnheitsrechts" geworden ist (so die Fragestellung auf S. 223). Zimmermann gliedert das von ihm gesichtete Praxismaterial zweifach, einmal für alle völkerrechtlichen Verträge nach den betroffenen Sukzessionsformen (S. 225-450), sodann für alle Sukzessionsformen in bezug auf besondere Vertragsarten (S. 451-751). Diese Vorgehensweise überzeugt vor dem Hintergrund der Praxis, die vielfach spezifische Sukzessionsregime für bestimmte Vertragskategorien entwickelt, widerspricht aber dem Konzept der Konvention, die in Art. 11, 12 lediglich die sog. radizierten Verträge hervorhebt.

Für die wenigen nach 1978 aufgetretenen Fälle von newly independent states zeigt sich, daß die Praxis weitgehend den Regeln in Teil III der Konvention gefolgt ist, so daß Zimmermann diese für den kolonialen Sonderfall als Völkergewohnheitsrecht einstuft (S. 233). Die Staatenvereinigung wird in der Konvention als einheitlicher Tatbestand behandelt, ohne zwischen Inkorporation und Fusion zu unterscheiden. Die Praxis trifft diese Unterscheidung allerdings sehr wohl, wie die Fälle Deutschlands und des Jemen zeigen: Ersterer orientierte sich grosso modo an den klassischen Inkorporationsregeln (Erstreckung der Verträge des fortbestehenden Staates, Erlöschen der Verträge des beitretenden Staates), während die jemenitische Vereinigung die in Art. 31 der Konvention vorgesehene allseitige Kontinuität nachvollzog (S. 287 f.). Für die Dismembration stehen Zimmermann nach der von ihm getroffenen Klassifizierung mit dem Zerfall Jugoslawiens und der Tschechoslowakei zwei neuere Fälle zur Verfügung. Die Durchsicht der Staatenpraxis bringt das - durchaus überraschende - Ergebnis, daß alle beteiligten Staaten die Regel der automatischen Universalsukzession grundsätzlich akzeptiert haben, so daß Art. 34 der Konvention insoweit als Völkergewohnheitsrecht anzusehen ist (S. 367 f.). Für den Tatbestand der Abspaltung gilt dies allerdings nicht. Hier zeigt sich anhand der Fälle Sowjetunion und Eritrea, daß der verbleibende Rumpfstaat selbstverständlich an die Altverträge gebunden bleibt, eine automatische Nachfolge aller abgespaltenen Neustaaten aber nicht akzeptiert wird (S. 429-431). In dieser Hinsicht entspricht Art. 34 der Konvention also nicht der gegenwärtigen Staatenpraxis. Für Zessionen schließlich sieht Zimmermann den gewohnheitsrechtlichen Grundsatz der beweglichen Vertragsgrenzen (Art. 15 der Konvention) in der Praxis bestätigt (S. 449), auch wenn die von ihm gewählten Beispiele Hongkong und Macao insoweit doch deutliche Eigenheiten aufweisen.

Von den besonderen Vertragsarten werden im Schrifttum stets die radizierten, also gebietsbezogenen Verträge (vor allem Grenzverträge) hervorgehoben, die in allen Fällen der Suk- 
zession fortgelten, also auch den Gebietsnachfolger binden sollen (so auch Art. 11, 12 der Konvention). Es überrascht daher wenig, daß Zimmermann dieses Regel auch in der jüngeren Staatenpraxis bestätigt findet (S. 484, 513 f.). In Anlehnung an radizierte Verträge will er auch völkerrechtliche Verträge zum Menschenrechtsschutz behandelt sehen (S. 572577); diese These vom automatischen Einrücken der Nachfolgestaaten wird allerdings durch das zuvor ausgebreitete Praxismaterial m.E. nicht gestützt. Mitgliedschaften in internationalen Organisationen gelten traditionell als sog. höchstpersönliche Bindungen eines Staates, die eine Staatensukzession nicht überdauern. Im wesentlichen bestätigt auch die Untersuchung Zimmermanns diese These, wenngleich in Vereinigungsfällen gelegentlich eine Fortführung der Mitgliedschaft im allseitigen Einvernehmen erfolgt (S. 659). Von den übrigen Sonderkonstellation, die dieser dritte Teil behandelt, sei noch die Funktionsnachfolge der Europäischen Gemeinschaften in Verträge ihrer Mitgliedstaaten herausgegriffen, die allerdings mit Recht klar von der Staatensukzession unterschieden wird (S. 712-718).

Im vierten Teil versucht Zimmermann anhand der vorangegangenen Ergebnisse eine Bewertung der Konvention von 1978. Da sie nur in begrenztem Maße die vor ihrer Abfassung verbreitete Staatenpraxis widerspiegelt, können nur wenige ihrer Bestimmungen wirklich als Kodifikation im eigentlichen Sinne gelten (S. 821-827). Auch die jüngere Praxis nach 1978 hat sich nur in einigen Fällen von den Bestimmungen der Konvention leiten lassen, die insoweit aber immerhin für sich in Anspruch nehmen könne, das Recht der Staatensukzession erfolgreich weiterentwickelt zu haben (S. 830-833). Insgesamt sei die Konvention trotz ihrer Mängel wenigstens als Leitlinie für künftige Sukzessionsfälle geeignet (S. 843).

Die Arbeit ist keine leichte Lektüre. Das buchhalterische Element, das derartigen „Sammelarbeiten“ eigen ist, läßt einen eleganten Schreibstil wahrscheinlich nicht zu. In der Sache aber gebührt dem Verfasser große Anerkennung dafür, einen schier unüberschaubaren Berg an Material gesichtet und wissenschaftlich aufbereitet zu haben. Diese mühselige Fleißarbeit, die man selten nur bei Habilitationsschriften findet, macht das Recht der Staatensukzession für den Bereich der völkerrechtlichen Verträge in seinen Rechtsfolgen zugänglich und damit praktisch anwendbar. Wenn schon nicht die Wiener Konvention von 1978, so bringt doch die Arbeit von Zimmermann das Völkerrecht ein gutes Stück voran.

Oliver Dörr, Berlin 


\section{Stefan Schuppert}

\section{Neue Steuerungsinstrumente im Umweltvölkerrecht am Beispiel des Montrealer Protokolls und des Klimaschutzrahmenübereinkommens}

Kosteneffektivität und Innovationswirkungen als Grundsätze in internationalen Verträgen Beiträge zum ausländischen öffentlichen Recht und Völkerrecht, Band 131

Springer Verlag, Berlin u.a., 1998, 297 S., DM 128,--

Kaum ein Bereich des internationalen Rechts hat sich in den letzten Jahrzehnten so rasant entwickelt wie das Umweltvölkerrecht. An die Stelle der Abgrenzung zwischenstaatlicher Kompetenzbereiche durch bilaterale Abkommen und das traditionelle Instrumentarium der Rechtsdurchsetzung traten multilaterale Übereinkommen zum Schutz staatengemeinschaftlicher und globaler Umweltmedien, die vor allem auf dem Sektor der Erfüllungssicherung eine Reihe an Innovationen mit sich brachten. Drei Aspekte dieser jüngsten Entwicklungen sind Gegenstand der anzuzeigenden Untersuchung: neue Steuerungsinstrumente ökonomischer Art, die vom nationalen Recht herkommend Eingang in das Völkerrecht gefunden haben, die Verwirklichung von Kosteneffektivität sowie von Innovationsförderung als Grundsätze und Ziele völkerrechtlicher Verträge.

Den Hintergrund der Untersuchung bilden drei völkerrechtliche Vertragsregime: Den größten Anteil nimmt das Wiener Übereinkommen zum Schutz der Ozonschicht ein, das auf das Jahr 1985 zurückgeht, durch das Montrealer Protokoll von 1987 ergänzt und in seinen Pflichtenstrukturen inzwischen fünf nachfolgenden Anpassungen unterworfen wurde. Neueren Datums ist das UN-Klimaschutzrahmenübereinkommen von 1992, das im Kyoto-Protokoll seine jüngste Ergänzung gefunden hat, die der Verfasser freilich nicht mehr hat berücksichtigen können. Während die vorgenannten Verträge solche mit universeller Verbreitungstendenz sind, gilt mit einem im Rahmen der UN-Wirtschaftskommission für Europa (ECE) entstandenen Vertrag ein weiteres Augenmerk des Verfassers einer regionalen Abmachung, dem Übereinkommen über weiträumige grenzüberschreitende Luftverschmutzung von 1979 mit seinem Protokoll von 1994. Der Schwerpunktbildung der Arbeit entsprechend soll im folgenden allein auf die ersten beiden der drei vorgenannten Regime eingegangen werden.

In dem der Einführung folgenden, zweiten Abschnitt wendet sich der Verfasser dem Wiener Übereinkommen und dem Montrealer Protokoll zu. Als wesentliche Neuerung kennzeichnet dieses Vertragsregime seine entsprechend dem Prinzip der ,common, but differentiated responsibilities“ unterschiedlich ausgestalteten Verpflichtungen beim Ausstieg aus ozonschädigenden Stoffen für Industriestaaten auf der einen und Entwicklungsländer auf der anderen Seite. Ihrer Ausgestaltung nimmt sich der Verfasser umfassend an (S. 36-135). Ein ökonomisches Steuerungsinstrument auf internationaler Ebene, das auf dem Gedanken der Kosteneffektivität beruht, stellen die Vorschriften über die gemeinsame Erfüllung von Vertragspflichten dar. Da in der Praxis jedoch eine Verlagerung von Produktion oder Verbrauch zwischen den Vertragsparteien nicht stattgefunden hat, sind entsprechende Vorschriften bislang Theorie geblieben, wie Schuppert zutreffend feststellt (S. 148). Ein weite- 
res, dem Regime immanentes völkerrechtliches Steuerungsinstrument ist schließlich die Beschränkung des internationalen Handels mit Nichtvertragsstaaten, wie sie Art. 4 Montrealer Protokoll vorsieht. Die Frage der Völkerrechtskonformität dieser Maßnahme hat jedoch durch die Nichtannahme des Berichts des GATT-Streitbeilegungspanels zum USmexikanischen Thunfischstreit an Brisanz verloren, da der Panel-Bericht keine Verbindlichkeit erlangte. Schließlich, darauf weist der Verfasser zutreffend hin (S. 159), enthalten andere umweltschützende Verträge des Völkerrechts keine entsprechenden Vorschriften über Handelsbeschränkungen. Die weitere Diskussion dieser Maßnahme stellt mithin eine vor allem akademische Übung dar.

Auch das Klimaschutzrahmenübereinkommen der Vereinten Nationen von 1992 weist direkte und indirekte Steuerungsinstrumente auf und enthält Regelungen, die auf den Grundsätzen von Kosteneffektivität, Effizienz und Innovationsförderung beruhen. Auf dem Gedanken der Kosteneffektivität fußt das hier vereinbarte Institut der Gemeinsamen Durchführung (sog. ,joint implementation“), das aber auch unter dem Aspekt der Innovationsförderung beachtenswert ist. Nach der Berliner Konferenz der Vertragsstaaten kam beiden Grundsätzen jedoch nur noch beschränkte Reichweite zu. Ihre Stellung ist vielmehr allein eine sekundäre, denn sie sollen durch eine bessere Nutzung finanzieller Mittel und den verstärkten Einsatz technologische Innovationen gestatten, die primären ökologischen Prinzipien umzusetzen (S. 173-239). Leider konnte das Kyoto-Protokoll vom Verfasser nicht mehr berücksichtigt werden. Von seinen Steuerungselementen hätte im vorliegenden Kontext insbesondere der Mechanismus des ,emissions trading“ Aufmerksamkeit verdient. Die von Stefan Schuppert vorgelegte Arbeit wurde im Sommer 1997 von der Rechtswissenschaftlichen Fakultät der Christian-Albrechts-Universität Kiel als Dissertation angenommen, betreut hat sie der Völkerrechtslehrer Professor Rüdiger Wolfrum. Im Rahmen des innovativen Ansatzes zeichnet sich die Untersuchung vor allem durch eine überzeugende Aufarbeitung des magischen Spannungsfeldes „Wissenschaft - Recht - Wirtschaft“ aus. Die Untersuchung beruht nicht nur auf den global bzw. regional wichtigsten Verträgen des klimaschützenden Umweltvölkerrechts, sondern berücksichtigt namentlich am Beispiel Deutschlands und der USA auch ihre Umsetzung in den innerstaatlichen Rechtsbereich. Dabei erschöpft sich die Darstellung nicht in der Erörterung (wissenschafts-)theoretischer Positionen, sondern unternimmt eine umfassende Analyse völkervertragsrechtlicher Pflichten, der sie begleitenden Praxis und der einschlägigen ökonomischen Hintergründe. Alles in allem eine überaus begrüßenswerte Publikation, an der die weitere Diskussion des Themas ,environmental economics“ nicht wird vorübergehen können.

Sebastian M. Seidel, Bochum 


\section{Jutta Kramer}

\section{Apartheid und Verfassung}

Das Staatsrecht als Instrument der Rassentrennung und ihrer Überwindung in Südafrika Nomos Verlagsgesellschaft, Baden-Baden, 2001, 247 S., DM 88,--

Die weitreichenden Veränderungen, denen die Republik Südafrika seit der richtungsweisenden Rede des letzten weißen Präsidenten Fredrik Willem de Klerk zur Parlamentseröffnung Anfang Februar 1990 unterliegt, haben hierzulande eine Vielzahl von Untersuchungen zu den unterschiedlichsten Aspekten eines sehr spannenden juristischen, politischen und gesellschaftlichen Transformationsprozesses angeregt. Einen weiteren Beitrag zur wissenschaftlichen Aufarbeitung von Apartheid und ihrer Überwindung liefert die bei Nomos in der Reihe „Recht und Verfassung in Südafrika“ erschienene Dissertation von Jutta Kramer.

Apartheid ist von Herrmann Vogt als ,lückenloses Rechtssystem mit dem Ziel der vollständigen Trennung der Rassen“ bezeichnet worden (Apartheid und Unterentwicklung - Möglichkeiten und Strategien gesellschaftlicher Veränderung in Südafrika, Frankfurt am Main, 1982, S. 10). Die Verfasserin identifiziert dieses System als wesentliches Herrschaftsinstrument der weißen Bevölkerungsminderheit und zeichnet mit ihrer nach territorialen, ethnisch-nationalen, personalen und funktionalen Gesichtspunkten geordneten Arbeit ein ausführliches Bild der fast fünf Jahrzehnte währenden gesetzlichen Rassentrennung in Südafrika. Ihre Bestandsaufnahme zeigt, dass das Normenspektrum des von der National Party (NP) sukzessive errichteten Apartheidregimes in der Tat nahezu lückenlos war; es reichte von Gesetzen zur Verwirklichung der alltäglichen ,petty apartheid“ (etwa zur Einrichtung von getrennten öffentlichen Verkehrsmitteln, Bibliotheken, sanitären Einrichtungen oder Stränden) über die Verankerung ,mittlerer“ Apartheid (etwa Einschränkungen von Freizügigkeit, Siedlungsfreiheit, Berufsfreiheit und politischen Rechten der schwarzen Bevölkerungsmehrheit im ,weißen“ Südafrika) bis hin zur Aufteilung des Landes nach ethnischen Kriterien durch die Ausweisung separater Siedlungsgebiete (,homelands“), entsprechende Zwangsumsiedlungen großer Bevölkerungsteile, die Entwicklung einer begrenzten Selbstverwaltung und - in vier Fällen - sogar die staatliche Ausgrenzung dieser Gebiete durch die Einführung einer formellen Unabhängigkeit im Rahmen der sogenannten „grand apartheid“. Die aufwendige Dokumentation dieser rechtlichen Infrastruktur konzentriert sich dabei nicht nur auf die Regierungszeit der NP; die Verfasserin macht deutlich, dass die ersten Schritte zu einer gesetzlich fundierten Rassentrennung bereits unter der britischen Herrschaft am Kap eingeleitet worden sind. Die geistigen Ursprünge von Apartheid lassen sich sogar bis in das 18. Jahrhundert zurück verfolgen.

Das Anliegen der Arbeit, ,dieses Rechtsgeflecht so vollständig wie möglich unter verfassungsrechtlichen und verfassungstheoretischen Aspekten darzustellen“ (S. 17), hat allerdings auch eine Kehrseite. Da die gesetzliche Rassentrennung in Südafrika tatsächlich fast jeden Lebensaspekt (bis hin zur sexuellen Selbstbestimmung) erfasste, hätte eine vertiefte Untersuchung einiger exemplarischer Bereiche der Verfasserin mehr Raum gegeben, politi- 
sche und gesellschaftliche Hintergründe sowie die praktischen Auswirkungen der von ihr angesprochenen Gesetze und Verordnungen ausführlicher darzustellen und die Instrumentalisierung von Rechtsetzung zu Zwecken des Machterwerbs und der Absicherung von Herrschaft hervorzuheben. So beschränkt sich die Arbeit stellenweise auf die Zusammenfassung der einschlägigen gesetzlichen Regelungen, ohne diese durch Einblicke in die jeweilige Parlaments-, Verwaltungs- und Rechtsprechungspraxis zu unterfüttern. Ein weiteres Problem der Arbeit liegt in der Gliederung des umfangreichen Materials. Zutreffend weist die Verfasserin darauf hin, dass eine rein chronologische Ordnung wichtige Zusammenhänge innerhalb der vier thematischen Schwerpunkte der Untersuchung (,Apartheid und Staatsgebiet“, „Apartheid und Staatsvolk“, „Apartheid und Bürgerrechte“ und „Apartheid und Staatsgewalt“") zerrissen hätte; die Alternative, nach einem einführenden Gesamtüberblick in jedem Abschnitt erneut den Bogen von den englischen Kolonien und burischen Republiken über die Verfassungen von 1909, 1961, 1983 und 1993/1996 zu schlagen, führt jedoch zu einer Reihe von Wiederholungen (beispielsweise zum Aufbau des Drei-Kammer-Parlaments von 1983 und zur Geschichte der , unabhängigen' Gebiete Transkei, Bophuthatswana, Venda und Ciskei) und erschwert es dem Leser zudem, sich ein zusammenhängendes Bild der einzelnen (jeweils durch spezifische außen- und innenpolitische Faktoren gekennzeichneten) Phasen in der Entwicklung von Apartheid zu machen. Zugleich ein Problem der Gliederung wie auch einer stofflichen Begrenzung bildet schließlich die bereits im Untertitel der Arbeit angedeutete Differenzierung zwischen dem Staatsrecht als Instrument der Rassentrennung und ihrer Überwindung. Die Verfasserin behandelt die einleitend aufgeworfene Frage, ob die seit Beginn der 90er Jahre eingeschlagene Verfassungsentwicklung einen wirksamen Beitrag zur Beseitigung von Apartheid leisten kann (S. 20), nicht immer als eigenständigen Untersuchungsgegenstand. So wird etwa die neue Verfassungslage im Abschnitt „Apartheid und Bürgerrechte“ lediglich im Rahmen einer Zusammenfassung dargestellt (S. 119) und die Bewertung der beiden Verfassungen von 1993 und 1996 im abschließenden analytischen Teil auf einen Unterabschnitt zum Kapitel „Apartheid als Prozeß gesellschaftlicher Desintegration“ (S. 209) beschränkt.

Dabei stellt die heutige Verfassungsordnung - wie Kramer an dieser Stelle auch zu Recht bemerkt - eine ,herausragende politische und gesellschaftliche Integrationsleistung dar, die in diesem Jahrhundert ihresgleichen sucht“ (S. 210) und über die der Leser gerne mehr erfahren hätte. Der mehrstufige Verfassunggebende Prozess, die Einfügung sogenannter „sunset clauses“ (zeitlich begrenzte Übergangsbestimmungen, etwa zur Bildung einer ,Regierung der Nationalen Einheit' unter Beteiligung aller wesentlichen politischen Kräfte), die Gewährung von grundrechtlichen Ansprüchen gegenüber dem Staat und die verfassungsrechtliche Absicherung von Programmen zur Beseitigung der gesellschaftlichen Folgen gesetzlicher Rassendiskriminierung (,affirmative action“) sind nur einige Beispiele für die Bedeutung, die das Staatsrecht bei der Bewältigung ethnischer Konflikte spielen kann, und bilden damit wichtige Gegenstücke zu dem von Kramer als „Verfassungsunrecht“ (S. 201) eingestuften Prozess gesellschaftlicher Desintegration unter weißer Alleinherrschaft. Dieser gezielte Einsatz von Staatsrecht zur Überwindung von Rassentrennung 
hätte eine ausführlichere Darstellung verdient (möglicherweise auch zulasten der für das Thema der Untersuchung weniger wichtigen Beschreibungen von Senat und National Council of Provinces, der Verteilung der Gesetzgebungskompetenzen und -verfahren sowie der Stellung des Staatspräsidenten), zumal die Umsetzung der genannten Instrumente bis heute nicht ohne rechtliche und praktische Probleme verläuft.

Die rechtstheoretische Deutung des Apartheidregimes ist nach der umfangreichen Darstellung seiner gesetzlichen Grundlagen leider vergleichsweise knapp ausgefallen. Trotzdem gelingt es der Verfasserin, die verschiedenen Phasen in der Entwicklung von Apartheid von der an praktischen Gesichtspunkten orientierten indirect rule der Briten über die einem übersteigerten Nationalismus entspringende Politik der Rassendiskriminierung in der Kernzeit burischer Herrschaft bis hin zu den ökonomischen Zwängen folgenden (aber weitgehend „kosmetischen“) Reformversuchen der Regierung von Pieter Willem Botha - in ihren wesentlichen Grundzügen zu charakterisieren. Dabei belegt die zum tieferen Verständnis dieser Zeit wichtige Erörterung der verschiedenen verfassungspolitischen Rechtfertigungsversuche, dass die gesetzliche Rassentrennung angesichts der fehlenden demokratischen Grundlage des Regimes und (selbst bei Gewährung entsprechender Partizipationsmöglichkeiten in den homelands) der ökonomischen Abhängigkeit der schwarzen Bevölkerungsmehrheit vom weißen Südafrika nicht zu legitimieren war. Unter Rückgriff auf die von Ernst Fraenkel für den Nationalsozialismus entwickelte „Theorie des Doppelstaates“ zeigt die Verfasserin, dass in Südafrika vielmehr zwei die Gesellschaft nach rassischen Merkmalen kategorisierende Systeme errichtet wurden. Während die privilegierte weiße Bevölkerungsminderheit in einem von Gewaltenteilung, Bindung der öffentlichen Gewalt an Gesetz und Recht, demokratische Teilhabe und Rechtsschutz durch unabhängige Gerichte gesetzten Rahmen (,Normenstaat“) lebte und lange Zeit nur mittelbar durch die Auswirkungen der Rassentrennung getroffen wurde (etwa durch die alle Bevölkerungsteile beeinträchtigenden Ausnahmezustände oder wechselseitig wirkende Verbote, etwa in Bezug auf ,Mischehen'), war insbesondere die schwarze Bevölkerungsmehrheit zahllosen diskriminierenden Sonderregelungen und einer behördlichen Willkür ausgesetzt, die nur selten durch die Justiz über die Anwendung des ,farbenblinden' Common Law begrenzt wurde („Maßnahmenstaat“). Das Staatsrecht - so das Fazit nach der Lektüre dieser thematisch interessanten Arbeit - war damit ein überaus wirksames Mittel zur Einführung und Aufrechterhaltung einer mit den Maßstäben eines modernen, demokratischen Verfassungsstaates unvereinbaren Herrschaft einer Minderheit in einer ethnisch definierten Gesellschaft; es erscheint heute aber als ein ebenso wirksames Instrument, die Folgen dieser gesetzliche Rassentrennung auf friedlichem Wege zu überwinden.

Jörg Fedtke, Hamburg 


\section{Ulrich Wehner}

\section{Der Mercosur}

Rechtsfragen und Funktionsfähigkeit eines neuartigen Integrationsprojektes und die Erfolgsaussichten der interregionalen Kooperation mit der Europäischen Union Nomos-Verlag, Baden-Baden, 1999, 253 S., DM 64,--

Man muß nicht gleich Simón Bolívar zitieren mit dem Ausspruch, es sei leichter, das Meer zu pflügen als eine lateinamerikanische Gesellschaft zu schaffen, um die Schwierigkeiten einer Integration auf dem Subkontinent darstellen zu können. Neuere Untersuchungen setzen an bei den Versuchen, die seit rund 40 Jahren unternommen werden, um dort zumindest eine Freihandelszone zu errichten.

So beginnt auch Ulrich Wehner in seiner hier vorzustellenden Dissertation von 1998 mit einem kurzen Überblick über die ersten Ansätze einer regionalen Integration durch die Asociación Latinoamericana de Libre Comercio (ALALC), die Asociación Latinoamericana de Integración (ALADI) und den Andenpakt, später dann umbenannt in Andengemeinschaft (bestehend aus Bolivien, Ekuador, Kolumbien, Peru und Venezuela). Ähnliche Versuche der Gemeinschaftsbildung in Mittelamerika und der Karibik werden nur kurz gestreift.

Alle diese Integrationsansätze sind in den 80er Jahren nach Ansicht von Wehner gescheitert bzw. stagnierten auf Grund anscheinend unüberbrückbarer Differenzen bereits bei der Schaffung von Freihandelszonen zwischen den Mitgliedsländern. An weitere ambitioniertere Schritte wie die Errichtung einer Zollunion, eines Gemeinsamen Marktes sowie, quasi als Endstufe der Integration, einer Wirtschafts- und Währungsunion war gar nicht mehr zu denken. Modellhaft für diese Versuche war, speziell für die Andengemeinschaft, die Europäische Union. Einher ging mit diesen Versuchen immer der Aufbau eines Institutionengebäudes, wodurch man - offensichtlich erfolglos - einen integrativen Anschub erwartete. In den 90er Jahren hat die Andengemeinschaft, nach der „Verlorenen Dekade“, wie die 80er Jahre häufig bezeichnet werden, einen erneuten Anlauf genommen, nunmehr mit einer Umstrukturierung seiner Institutionen und der weiteren Errichtung von, wie man glaubt, integrationsfördernden Einrichtungen.

Im Hauptteil seiner Arbeit beschreibt Wehner die Integrationsziele des Mercosur, seine Rechtsquellen, das Institutionengefüge sowie rechtsdogmatische und völkerrechtliche Aspekte dieses Integrationsmodells. Ausgangspunkt ist der Vertrag von Asunción vom 26.03.1991, in dem sich Argentinien, Brasilien, Paraguay und Uruguay darauf verständigt hatten, einen Gemeinsamen Markt mit dem Namen „Mercosur“ zu gründen.

Danach verdient hervorgehoben zu werden, daß der Mercosur, im Gegensatz zu allen anderen Integrationsansätzen, bewußt auf die Errichtung von starken Gemeinschaftseinrichtungen verzichtet. Das bedeutet, alle Entscheidungen müssen konsensual getroffen werden, und zwar immer auf Regierungsebene. Dies fördert zweifellos die Akzeptanz der Gemeinschaft in den Mitgliedstaaten. Wehner verkennt aber auch nicht die Kehrseite dieses Ansat- 
zes, nämlich „das mangelnde Vertrauen in die Kontinuität und Irreversibilität des Gesamtprojekts“.

Zunächst hat der interne Warenaustausch signifikante Zuwachsraten verzeichnet. Nicht genau feststellbar ist aber, ob dies durch den punktuellen Zollabbau erreicht wurde oder doch eher ein Phänomen der allgemein verbesserten Wirtschaftslage in den Mitgliedstaaten ist. Zukünftig will man eine lineare Senkung der internen Zölle vereinbaren. Hier wird sich allerdings erst zeigen, inwieweit die Regierungen bereit und willens sind, besonders sensible nationale Wirtschaftsbereiche einem ungeschützten Wettbewerb auszusetzen. Speziell zwischen Argentinien und Brasilien sind diesbezüglich schon erste Spannungen sichtbar geworden. Paraguay und Uruguay spielen dabei nur eine untergeordnete Rolle. Und dann wird auch das Problem auftauchen, ob man weiterhin die Streitschlichtung allein auf politischer Ebene austragen will oder ob nicht doch unabhängige Gemeinschaftsorgane, sprich: Gerichte mit supranationalen Kompetenzen, erforderlich sind.

Wehner befaßt sich ausführlich mit der Kritik am Mercosur wegen seines fehlenden Institutionengefüges, einhergehend mit der geringen Neigung bei den Mitgliedstaaten, vom Prinzip des ,rein zwischenstaatlichen intergouvernementalen Integrationsansatz(es)“, abzurücken.

Dieser Ansatz ist insofern auch verständlich, da das - vermeintliche - Vorbild Europäische Union aufgrund der Ausgangslage und der wirtschaftlichen Rahmenbedingungen keinen exemplarischen Charakter für die Entwicklungsländer auf dem Subkontinent haben kann. Zum anderen bietet die in jeder Hinsicht defizitäre Andengemeinschaft, die dem EU-Vorbild anscheinend nacheifern will, wenig Anlaß zur Übernahme dieses Modells.

Dennoch bleibt abzuwarten, ob tatsächlich, wie Wehner es tut, vom Mercosur als einem Integrationsmodell sui generis gesprochen werden kann. Denn noch immer ist die Freihandelszone als Vorstufe zu dem geplanten Gemeinsamen Markt nicht ausreichend verwirklicht, um aus dieser Gemeinschaft schon ein Modell konstruieren zu können.

Jürgen Saligmann, Bonn

Klaus Bodemer / Heinrich-W. Krumwiede / Detlef Nolte / Hartmut Sangmeister (Hrsg.)

Lateinamerika-Jahrbuch 2000

Institut für Iberoamerika-Kunde, Hamburg

Vervuert-Verlag, Frankfurt/Main 2000, 312 S., DM 28,--

Auch der neunte Band dieser erst 1992 begonnenen Reihe, die übrigens ein gelungenes Beispiel dafür ist, dass auch relative Spätentwickler sich erfolgreich auf dem Markt etablie- 
ren können ${ }^{1}$, überzeugt mit seiner wiederum gelungenen Kombination von gediegener Analyse und faktenreicher Berichterstattung. Gegliedert wie seine Vorgänger ${ }^{2}$, widmet er sich eingangs mehreren gesamtlateinamerikanischen Themen in Aufsatzform und referiert sodann die Entwicklungen einschließlich statistischer Grunddaten aller - nach (Sub-) Regionen zusammengefasster - Länder südlich des Rio Grande. Dass er sich für die „Kleinund Kleinststaaten im Karibischen Raum“ jeweils mit tabellarischen Darstellungen ihrer demographischen, sozialen und wirtschaftlichen „Kennziffern“ begnügt, leuchtet ein. Indes fragt sich, warum an diesem Katzentisch auch die Dominikanische Republik Platz nehmen muss - als Land immerhin spanischer Zunge doch eigentlich bei den Grossen platzberechtigt, wo sich selbstverständlich Cuba und sogar ihr frankophoner Insel-Nachbar Haiti finden.

Berichtszeitraum ist das Jahr 1999, was die meisten statistischen Tabellen bei 1998 enden lässt. Den Nutzen des Bandes als Nachschlage-Werk wie auch den Wert seiner Textbeiträge mindert dies jedoch nicht im geringsten. Die für die Leserschaft dieser Zeitschrift vor allem interessanten politischen Verhältnisse (Parteien, Zusammensetzung von Regierungen, Kräfteverhältnisse in den Parlamenten pp.) finden sich ohnehin durchweg bis Juli/August 2000 nachgeführt.

Die Themen des ersten Teils entstammen heuer ausschließlich lateinamerikanischer Innenpolitik. Der Aufmacher von Detlef Nolte, Hamburg („Lateinamerikas Parteien zwischen Volatilität und Beharrung") gibt eine höchst informative tour d'horizon zu Standorten und Entwicklungen iberoamerikanischer Parteiensysteme in den 80er und 90er Jahren. Vor dem Hintergrund von 82 Schrifttumstiteln und 5 Statistiken zu Akzeptanz, „Flüchtigkeit“ und Vitalität der Parteien im lateinamerikanischen Vergleich nimmt der Autor die politikwissenschaftliche Bewertung der demokratischen Funktion politischer Parteien im fast sinuskurvigen Wechsel von Grabgesang (in den Jahren der Diktatur) und Hosianna (seit den Demokratisierungsschüben) zum Anlass, nachhaltigen Forschungsbedarf zur Relation zwischen Parteien- und Systemqualität anzumelden. Manfred Wöhlcke, Ebenhausen (,Das Bevölkerungswachstum in Lateinamerika") reklamiert zu Recht für den - überraschend vielerorts unterschätzten - Gegenstand seiner Analyse einen der obersten Plätze auf der politischen Agenda in Gegenwart und Zukunft. Die regionale Problemdimension verdeutlicht insbesondere sein Vergleich, was es sogar für ein Land wie die Bundesrepublik Deutschland bedeuten würde, wüchse hier die (eigene) Bevölkerung um jährlich rund 1,5 Mio. Menschen - etwa die Einwohnerzahl Münchens. Regionale Entwicklung, Altersstruktur, soziale Systeme, Urbanisierung, Umwelt und Ressourcen, demokratische Stabilität, sicherheitspolitische Gewichtsverlagerungen und internationale Migration sind denn

Unter den Jahrbüchern des Deutschen Übersee-Instituts Hamburg erscheinen das Japan-Jahrbuch schon seit 1977, das Afrika- und das Nahost-Jahrbuch seit 1988; jüngeren Datums sind lediglich das Korea-Jahrbuch (1996) und das Indien-Jahrbuch (1998).

Vgl. die Rezension des 1996er Bandes von Frank Niemeyer in VRÜ 30 (1997), S. 425 ff. 
auch die Eckpunkte, an welchen sich der Autor orientiert, wobei namentlich der letztgenannte Aspekt hierzulande dürfte aufhorchen lassen.

Ein in der Sache nicht weit entferntes Feld bearbeitet Susana Sottoli, Asunción (,Sozialpolitik in Lateinamerika im Zeichen von Marktwirtschaft und Demokratie“). Die auch dort innert der letzten 15 Jahre favorisierte Entthronung des Staates zugunsten des Marktes mittels Deregulierung, Dezentralisierung, Privatisierung lasse tradierte Sozialpolitiken umschlagen in stärker neoliberal geprägte Ansätze. Frühere sozialpolitische Zielvorstellungen - „Solidarität, Verteilungsgerechtigkeit, Kollektivismus, Egalitarismus“ - wichen neuen Wertigkeiten wie „Subsidiarität, Individualismus, Selbstverantwortung, Voluntarismus, Residualismus, individuelle Freiheit, Leistung, Effizienz“. Dies korrespondiere mit einem auch entwicklungspolitischen Paradigmenwechsel, so beispielsweise im Bereich wirtschaftlicher Regelungsmuster weg vom starken Staatsinterventionismus, mittels dessen der Staat fungiert habe als “(...) wirtschaftlicher Allokateur von Ressourcen und als Hauptentwicklungsträger“ hin zum „Markt als zentralem Allokationsmechanismus, Rückzug des Staates aus der Wirtschaft bzw. subsidiäre Rolle des Staates“. Die Autorin lässt keinen Zweifel aufkommen, dass hier nicht alles, was theoretisch glänzt, auch praktisch Gold ist: So dienten Privatisierung und Dezentralisierung nicht selten lediglich einer Entlastung des Zentralstaates, ohne dass zugleich die für eine erfolgreiche Aufgaben-Delegation notwendigen persönlichen, technischen und finanziellen Voraussetzungen geschaffen würden. Deshalb, so das Fazit, sollten privatisierten wie dezentralisierten Sozialleistungen distributive und sozialausgleichende Elemente implementiert werden. Auch dies ist gewiss leichter gesagt als getan; aber richtig ist allemal, dass Dezentralisierung nicht schlichtweg vertikales out-sourcing sein kann, wobei übrigens - aber das richtet sich an die DezentralisierungsDebatte allgemein - man sich mitunter eine präzisere Begrifflichkeit wünscht, denn etliches, was in jener Debatte „Dezentralisierung“ heißt, wäre in Wahrheit, sofern lediglich die unmittelbare Staatsverwaltung vertikal entflochten werden soll, Dekonzentrierung.

Thematisch nahtlos schließt das Autoren-Duo Barbara Kloss-Quiroga / Axel Kroeger, Berlin / London, auf mit seinem Beitrag („Gesundheitssituation in Lateinamerika und Reformen im öffentlichen Gesundheitswesen"). Sie demonstrieren den auch von ihnen beobachteten Paradigmenwechsel zu neoliberalen Denkmustern in der lateinamerikanischen Sozialpolitik auf deren sensibelstem Sektor anhand allgemeiner Grundfaktoren wie vor allem Art und Umfang der Gesundheitsdienste, struktureller und funktionaler Reformbedarf, Rolle des Staates sowie gesundheitspolitische Strategien und Einflüsse internationaler Geberorganisationen wie Weltbank, WHO, Interamerikanische Entwicklungsbank (BID), Panamerikanische Gesundheitsorganisation (PAHO). Vor der Notwendigkeit, der gesamten Bevölkerung gleichen Zugang zur notwendigen gesundheitsdienstlichen Versorgung zu verschaffen und zu sichern, gewinnen solche gesundheitspolitischen Reformstrategien auch allgemein politisch-partizipative Dimensionen. Wie sich diese Strategien zur Effektivierung der gesundheitsdienstlichen Versorgung mittels Berücksichtigung aller im Gesundheitssektor tätigen Organisationen nach dem Grundsatz, so viel Privatisierung wie möglich, so viel dezentralisierte Staatlichkeit wie nötig, bislang konkret ausgewirkt haben, 
demonstrieren die Autoren anschließend an drei Fallbeispielen: Bolivien, Chile und Kolumbien. Die allen drei Ländern gemeinsame, normativ komplette Umstrukturierung traf bei der Delegation des Gesundheitswesens an die Gemeindeebene auf hier jeweils höchst unterschiedlich entwickelte administrative und personelle Gegebenheiten. Dabei hat es Bolivien mit seiner ohnehin für eine flächendeckende gesundheitsdienstliche Vernetzung wenig förderlichen Geographie auch und vor allem wegen seines beträchtlichen strukturellen Nachholbedarfs augenscheinlich am schwersten. Chile ist am anderen Ende des Spektrums bereits so weit, in Zusammenarbeit mit der PAHO ein Qualitätssicherungssystem für Krankenhäuser und ein aufwendiges Arzneimittelkontrollprogramm anzusteuern. Kolumbien wollte schon vor fünf Jahren mit seinen Reformen durch sein, stand 1999 mit der Umsetzung der Dezentralisierung aber noch immer bei erst 50\% seiner Departements und etwa 5\% seiner Gemeinden.

Was das Lateinamerika-Jahrbuch sodann in den folgenden einzelnen Länder-Berichten („Basisdaten - Kennziffern - Chronologien“) an äußerst gewissenhaft und sorgfältig ineinander gefügten Details zu politischen, wirtschaftlichen, sozialen Ereignissen und Vorgängen pro Land bietet, lässt kaum etwas zu wünschen übrig. Nicht zuletzt diese Berichte, die ohnehin quantitativ den Löwenanteil ausmachen, hat man im Blick, wenn man auch diesem Band verdientermaßen attestiert, eine profunde und weiterempfehlenswerte Informationsquelle $\mathrm{zu}$ sein nicht nur für diejenigen, denen die Verhältnisse in Lateinamerika professionales Forschungsobjekt, sondern auch für alle, denen sie aus anderen Gründen ans Herz gewachsen sind.

Karl-Andreas Hernekamp, Hamburg

Arne Rathjen (Hrsg.)

Neue Aspekte des Wahlrechts und des gewerblichen Rechtsschutzes in Brasilien und Deutschland

Schriften der Deutsch-Brasilianischen Juristenvereinigung, Band 27

Peter Lang Verlag, Frankfurt am Main, 1999, 137 S., DM 65,--

Der Herausgeber, der Berliner Rechtsanwalt Arne Rathjen, veröffentlicht in diesem Tagungsband die Beiträge zur 16. Jahrestagung der Deutsch-Brasilianischen Juristenvereinigung im Jahre 1997. Die ersten Beiträge von Prof. Sérgio Sérvulo da Cunha, Prof. Dr. Friedrich Müller und Prof. Dr. Wolf Paul widmen sich dem Themenkomplex Wahlrecht, die von Prof. Dr. Fritz Traub, Sibylle E. Schlatter, Gert Egon Dannemann und Juliana Viegas dem Themengebiet gewerblicher Rechtsschutz.

In einigen einleitenden Vorbemerkungen zu seinem Beitrag über der Wiederwahl des Präsidenten der Republik geht Sérgio Sérvulo da Cunha auf den generellen Aspekt der Wie- 
derwahl des Präsidenten in Lateinamerika ein, wobei sich der Autor dahingehend äußert, dass jede Wiederwahl der erste Schritt zu einer Diktatur oder mindestens ein Instrument zur Verzerrung des Wahlvorgangs darstellt. In diesem Zusammenhang wird auch die Historie der Wiederwahl von Präsidenten in den Staaten Lateinamerikas anhand von verschiedenen Zitaten dargestellt. Hieran schließt sich ein zweiter Abschnitt, welcher die relevanten Artikel der aktuellen brasilianischen Verfassung von 1988 nennt, die eine Wiederwahl des Präsidenten, der Gouverneure und Bürgermeister ausschließen. Danach wird das erste Scheitern eines Reformversuchs von 1994 und die damaligen Diskussionsbeiträge der Presse beschrieben. Schließlich geht der Autor auf die geplante Reform von 1997 ein. Dabei werden Zitate und Meinungen von Abgeordneten auch zu dem Thema ,der Preis der Wiederwahl“" erläutert und die Verfassungsmäßigkeit dieses Vorhabens beleuchtet. Zum Abschluss dieses Beitrags zitiert der Autor aus einem Kommentar zu den früheren kaiserlichen Anordnungen.

In seinem Beitrag zu aktuellen Legitimationsfragen des Wahlrechts in Deutschland und Brasilien erläutert Friedrich Müller zunächst Begriff und Legitimationsmodelle. Dabei beschäftigt sich der Autor mit der Bedeutung von „Legitimität“ und „Wahlrecht“ und geht auf die Unterschiede zwischen Wahlen und Abstimmungen ein. Im Laufe dieser Darstellung kommt Müller dann zu dem Schluss, dass die Ausdrücke Wahlen und Abstimmungen den Abstand zwischen direkter und indirekter, zwischen plebiszitärer und repräsentativer Demokratie markieren. Anschließend folgt eine Aufzählung der Gemeinsamkeiten hinsichtlich der beiden vorgenannten Begriffe in Deutschland und Brasilien.

In dem darauffolgenden Abschnitt 2 wird auf aktuelle Streitfragen eingegangen. Dabei werden hauptsächlich die unterschiedlichen Legitimierungsansätze innerhalb der repräsentativen Demokratien herausgearbeitet, wobei das deutsche Wahlrecht mit seiner ,personalisierten Verhältniswahl“" ausführlich, auch unter Beachtung des Entstehens von Überhangmandaten, erläutert wird. Schließlich widmet sich der Autor dann der inzwischen entschiedenen Streitfrage in Brasilien, ob die Vorschriften der Art. 82, $14 \S 5$ der Verfassung, wonach der Präsident nicht wiedergewählt werden darf, dahingehend geändert werden sollten, dass eine Wiederwahl des Präsidenten zulässig sein soll. Gerade diese Konstellation wird als Problem zur verfassungspolitischen Legitimität des Präsidenten erkannt und diskutiert. Hauptargument ist in dieser Diskussion der Begriff der politischen Kontinuität, die mit einer einzigen 4-jährigen Amtszeit ohne Möglichkeit zur Wiederwahl nicht gewährleistet ist. Als weiteres Hindernis zur verfassungspolitischen Legitimität wird erläutert, dass es bei der geplanten Verfassungsänderung Brasiliens, welche die Wiederwahl des Präsidenten ermöglichen soll, nicht um ein Einzelfallgesetz geht, das von Zeit zu Zeit notwendig sein kann, sondern dass es sich hier sogar um ein Einzelpersonengesetz auf Verfassungsebene handelt. Im Anschluss werden die Vorschriften des Grundgesetzes, welche die Änderung einzelner Artikel daraus regeln, ausführlich besprochen. Am Ende dieses Abschnitts geht der Autor dann noch kurz auf den Begriff der Legalität ein.

In einem letzten dritten Abschnitt über das Wahlverhalten und die Legitimität, wendet sich Müller einem Grundproblem zu, das mittlerweile in sehr vielen Ländern besteht. Hierbei 
handelt es sich um die abnehmende Repräsentativität der demokratisch gewählten Vertreter oder Staatsoberhäupter. Als im negativen Sinne herausragendes Beispiel werden die USA genannt, bei deren Wahlen für das Amt des Präsidenten oder zum Kongress nur noch 38\% bzw. 25\% der Wahlberechtigten von ihrem Wahlrecht Gebrauch gemacht haben. Als Schlussbemerkung stellt sich der Autor die Frage, ab welchem Grad der Nichtbeteiligung eine inhaltliche demokratische Legitimation nicht mehr möglich ist.

Der Beitrag „Die Wiederwahl des Präsidenten der Republik“ von Wolf Paul stellt eine Chronik der Verfassungsänderung hinsichtlich der Wiederwählbarkeit des Präsidenten von Brasilien dar. Dabei werden die relevanten Artikel der Verfassung für diese Reform übersichtlich gegenübergestellt und analysiert. Daran schließt sich eine zeitliche Abfolge der wichtigsten Etappen zur Ermöglichung der o.g. Reform an. Schließlich beschreibt der Autor eingehend das brasilianische System zur Verfassungsänderung. Hierbei werden die notwendigen Lesungen und Stimmenmehrheiten in beiden Kammern des Parlaments genannt und erläutert. Auch fehlt nicht ein Exkurs wie die Stimmenmehrheiten, teilweise durch Irregularitäten, zustande gekommen sind. Die schwerwiegendste ist die sog. „Stimmenkaufaffäre“, welche in ihrem gesamten Verlauf präzise und eindeutig von Paul beschrieben wird. In einem letzten Abschnitt beschäftigt sich der Autor dann eingehend mit dem Versagen der institutionellen Kontrollorganen, welche die o.g. Irregularitäten z.B. nicht durch eine Verfassungswidrigkeitsklage beim Obersten Bundesgericht oder einer Anfechtungsklage beim Obersten Wahlgericht geltend gemacht haben. In einer Anmerkung stellt Paul fest, dass bis zum Jahre 2002 eine dritte Amtszeit für den Präsidenten ausgehandelt werden könnte, da in den lateinamerikanischen Staaten die Verfassung als dispositives Recht angesehen werde.

Fritz Traub geht in seinem Aufsatz zum „Verbraucherleitbild im Wettbewerbsrecht“ zunächst auf die Frage ein, wer überhaupt das Schutzobjekt des Wettbewerbs- und Kartellrechts ist. Dem folgt eine intensive Auseinandersetzung mit dem Verbraucherleitbild aus Sicht der deutschen Rechtsordnung und anhand von Urteilen des EuGH zu diesem Thema. Dabei kommen aber auch Stellungnahmen zum Verbraucherleitbild aus der Lehre nicht zu kurz und werden beispielhaft dargestellt. Dabei stehen die wankelmütigen Urteile des EuGH in der Kritik, und Traub geht in diesem Zusammenhang auf eine Vorlage des Bundesverwaltungsgerichts vor dem EuGH gem. Art. 177 EWG-Vertrag (heute Art. 234 EGV) hinsichtlich eines lebensmittelrechtlichen Streits ein. Schließlich geht der Autor einen „Schritt zurück“ und erörtert sodann die Frage, ob die europäischen Instanzen sich überhaupt in die Frage des Verbraucherleitbildes einmischen dürfen oder nicht. Dabei kommt er zu dem Ergebnis, dass im Grundsatz zwar das Verbraucherleitbild den einzelnen Mitgliedstaaten überlassen bleibt, dies jedoch nicht ausschließt, dass der EuGH für gewisse Modelle Sympathien hegt oder nicht. Zur Begründung dieser Auffassung werde im Anschluss daran verschiedene Grundsatzurteile, wie das Cassis-Urteil aus dem Jahre 1979, die Entscheidungen Kohl/Ringelhan und Rennet S.A., sowie das Nissan-Urteil aus dem Jahre 1992, näher erläutert. Als weiteres Beispiel dafür, dass das Verbraucherleitbild des EuGH der mündige Verbraucher ist, wird die Mars-Entscheidung aus dem Jahre 1995 herangezogen. Diese 
wird ausführlich besprochen. In einem Schlussappell wendet sich der Autor gegen eine Veränderung des Verbraucherleitbildes des schutzbedürftigen Verbrauchers.

Sibylle E. Schlatter geht in der Einleitung ihres Beitrags zur wechselseitigen Ergänzung von Urheberrechtsschutz, gewerblichen Schutzrechten und Schutz gegen unlauteren Wettbewerb zunächst auf die Stellung des gewerblichen Rechtsschutzes und der Urheberechte in der Rechtsordnung ein und bestimmt die verfassungsrechtliche Grundlage. Im Anschluss werden in einem zweiten Abschnitt der Gegenstand und Umfang des Schutzes geistigen Eigentums erläutert. Dabei wird das Urheberrecht ausführlich besprochen und die Leistungsschutzrechte, welche den Anspruch auf das geistige Eigentum durchsetzen sollen, anhand von Beispielen erklärt. Das Patentrecht findet ebenfalls große Beachtung im Rahmen dieses Abschnitts. Als weitere Punkte werden noch der Gebrauchsmuster-, Geschmacksmuster- und Kennzeichenschutz definiert und deren Anwendungsgebiete, sowie Dauer des Schutzes besprochen. Der dritte Abschnitt erläutert die wechselseitigen Ergänzung des Immaterialgüterschutzes anhand der vorangegangenen Darstellungen und aktueller Rechtsprechung. Schließlich werden im darauffolgenden Abschnitt 4 der Gegenstand des Schutzes gegen unlauteren Wettbewerb dargestellt. Hierbei argumentiert die Autorin überwiegend mit Literaturmeinungen und geht weniger auf die Rechtsprechung zu diesem Thema ein. Der fünfte Abschnitt beschäftigt sich ausführlich mit dem wettbewerbsrechtlichen Leistungsschutz als Ergänzung des Immaterialgüterrechts. Dabei werden zunächst die Literatur- und Rechtsprechungsmeinungen aufgezeigt und detailliert erläutert, wobei die anerkannten Schutzlücken in dem Rechtsschutzsystem besondere Erwähnung finden. Daran schließt sich die Abgrenzung von unmittelbarer und nachschaffender Leistungsübernahme an, welche nach Meinung der Autorin Schwierigkeiten bereiten kann. Anschließend werden die Kriterien der Herkunftstäuschung und die wettbewerbswidrige Behinderung näher beleuchtet und anschaulich mit Beispielen erklärt. Am Ende des Beitrags widmet sich die Autorin noch prozessrechtlichen Fragen im Rahmen des wettbewerbsrechtlichen Leistungsschutzes.

Gert Egon Dannemann beschreibt in seinem Beitrag „Die neue brasilianische Gesetzgebung zum gewerblichen Rechtsschutz“ zunächst den politischen Werdegang dieser neuen Gesetzgebung von dem ersten Regierungsentwurf aus dem Jahre 1991 bis zur Unterzeichnung des Gesetzes über den gewerblichen Rechtsschutz, welches der Präsident 1996 unterzeichnete. Danach werden die grundlegenden Änderungen übersichtlich dargestellt. Gegenstand dieser Abhandlung sind die Patente und dazugehörige Verbote, sowie die Schutzfristen, welche anhand der maßgebenden Artikel des neuen Gesetzes erläutert werden. Dem schließt sich ein Abschnitt über Marken mit den wichtigsten gesetzlichen Änderungen, mit Erläuterungen der entscheiden Artikel des Gesetzes und der Schutzvorschriften an. In einem weiteren Punkt wendet sich der Autor den Rechtsverletzungen im Bereich des gewerblichen Rechtsschutzes zu, indem er Begriffe definiert und anhand des zivil- und strafrechtlichen Verfahrens die Konsequenzen der Zuwiderhandlungen darlegt. Zum Abschluss werden die Besonderheiten von Lizenz-, Technologie- und Franchise-Verträgen erläutert. 
In der Einleitung zu ihrem Beitrag über Technologieverträge gemäß dem neuen Gesetz über industrielles Eigentum gibt Juliana Viegas kurz die Geschichte der Gesetzgebung Brasiliens hinsichtlich des Technologietransfers und des Schutzes des industriellen Eigentums wieder. Gegenstand des zweiten Abschnitts ist dann die Rechtslage auf der Basis des Gesetzes Nr. 5772 vom 21. Dezember 1971 über das industrielle Eigentumsrecht. Dabei werden die damals entscheidenden Artikel aufgeführt, erläutert und durch die ergänzenden normativen Akte beschrieben. Einen entscheidenden Beitrag liefert ebenfalls die Darstellung der Umstände, unter welchen das Importverbot ausländischer Produkte im Jahre 1990 aufgehoben wurde und schließlich die Liste mit verbotenen Importprodukten 1991 gleichfalls abgeschafft worden ist. Im Anschluss daran werden die Folgen für die brasilianische Industrie beschrieben. Schließlich wendet sich die Autorin der aktuellen Rechtslagen gemäß dem neuen Gesetz über industrielles Eigentum aus dem Jahr 1997 zu. Dabei finden auch die nunmehr neuen Definitionen der Gesetzesbegriffe Eingang in die Betrachtung. Anhand eines Beispiels über die Bezahlung von Royalities für registrierte Marken werden die Unterschiede zur Gesetzeslage von 1991 und seit 1997 aufgezeigt. In der Abschlussbemerkung appelliert die Autorin an die nationale Kartellbehörde, dass es schön wäre, wenn diese den Technologietransfer weiter vereinfachen würde, um die Wettbewerbssituation brasilianischer Unternehmen auf den internationalen Märkten zu stärken.

Paulo B. Casella, São Paulo / Rainer W. Bauer, Bad Kreuznach

\section{Laura D. von Mandach}

\section{Recht und Gewalt}

Eine empirische Untersuchung zur Strafverfolgung in Brasilien

Forschungen zu Lateinamerika, Bd. 37

Verlag für Entwicklungspolitik Saarbrücken, Saarbrücken, 2000, 290 S., DM 52,--

Diese soziologische Arbeit (eine Züricher Dissertation) beschäftigt sich mit illegaler Gewaltanwendung aus dem staatlichen Bereich heraus. Die dabei nicht zu übersehende Enttäuschung der Schweizer Autorin (aufgewachsen in Brasilien) über die immerwährende violência auch in der wiederhergestellten Demokratie Brasilien ist abgeleitet aus umfangreichem Material, das sie in der Recherche (Analyse von Prozeßakten, 33 Experteninterviews, Besuch von Gerichtsverhandlungen) während eines eineinhalbjährigen Feldaufenthaltes und aus den Berichten von Amnesty International und Human Rights Watch ziehen kann. Untersucht wurde das brasilianische Strafverfolgungsmodell im Offizialverfahren mit seiner strikten Trennung von Vor- und Hauptverfahren (sistema do dupio inquérito), das eingehend geschildert wird. 
Dem gegenübergestellt wird die Strafrechtspraxis etwa mit dem faktisch ungleichen Zugang zur Justiz durch den strikten Anwaltszwang, für die unteren Einkommensschichten durch den im Rahmen eines Verfassungszusatzgesetzes 1994 eingeführten Pflichtverteidiger nur unzureichend abgemildert. Das polizeiliche Vorverfahren leidet u.a. daran, daß die ohnehin überlasteten und unterbezahlten Polizisten einen Teil ihrer Zeit für einen Nebenerwerb benötigen. Ermittelt wird regelmäßig zu Lasten des Angeschuldigten. Dem offiziellen Vorverfahren geht häufig eine bürokratisierte informelle, gesetzlich nicht vorgesehene Vorabklärung voraus.

Die Staatsanwaltschaft, ein eigener abgeschlossener Zirkel, beteiligt sich an der polizeilichen Ermittlung nur selten. In ihrer Stellung zwischen der ermittelnden Polizei und dem entscheidenden Gericht wird sie kaum wahrgenommen. Die Kompetenzausweitung für die Staatsanwaltschaft in Art. 129 Abs. 3 der Verfassung wird einfachgesetzlich letztlich nicht umgesetzt.

Die Macht der elitären Richterschaft dagegen ist groß und wird nach Bedarf jenseits der Verfassung umgesetzt. Die in ihr verankerte Unschuldsvermutung wird durch den richterlichen Hinweis an den Angeklagten, wonach sein Schweigen zu seinen Ungunsten ausgelegt werden könne, unterlaufen. Ein durchgehender Verfassungsskeptizismus (carta agoniza) ist unter Juristen allenthalten zu konstatieren (vgl. Intern. Seminar in Porto Alegre Nov. 1998, Mitt. Deutsch-Bras. Juristenvereinigung, März 1999, S. 25 ff.).

Die Arbeit schließt ab mit einem Kapitel über „Staatliche Gewalt außerhalb des Strafverfahrens“ einschließlich der Darstellung hinlänglich bekannter Gewaltanwendungen bei ländlichen Grundbesitzkonflikten.

Für die Wahrnehmung der Rechtswirklichkeit des brasilianischen Strafverfolgungssystems ist diese Arbeit mit ihrem sorgfältig aufbereiteten umfänglichen Material unverzichtbar.

Gerhard Scheffler, Hamburg

Werner Draguhn (Hrsg.)

Indien 2000

Institut für Asienkunde, Hamburg, 2000, 442 S., DM 48,--

Man braucht sich keine Sorgen zu machen, dass einem jährlich erscheinenden Berichtsband über Indien eines Tages der Stoff ausgeht. „Indien 2000“ berichtet wieder über das Menschenkonglomerat am Indischen Ozean mit seinen in- und externen Chancen und Problemen.

Formal ist 1999 und gegebenenfalls das erste Quartal 2000 einbezogen. Das Gros der Beiträge liefert datenunabhängige Befindlichkeiten und Entwicklungstendenzen. 
Innenpolitisch war 1999 bedeutsam, dass die südindische, mit schweren Korruptionsvorwürfen belastete (zum Teil in erster Instanz verurteilte) Frau Jayalalitha zwar die Zentralregierung in Delhi durch Zurückziehen ihrer zwei Minister stürzte, die Neuwahlen aber den alten Premier Vajpayee kräftigten. Dieser führt wieder die Vielparteienkoalition NDA (National Democratic Alliance), diesmal mit absoluter Parlamentsmehrheit. J. Beltz stellt die wesentlichen, teilweise atemberaubenden Ereignisse knapp und klar dar. D. Rothermund beschreibt Werdegang und eventuelle Aussichten des Ministerpräsidenten Chandrababu Naidu, Ministerpräsident des südindischen Landes Andhra Pradesh, und J. Eckert die gewalttätige hinduistische „Bewegung“ Shivsena im westlichen Bundesstaat Maharashtra, die aus lokalen Zellen aufbauend in militanter Weise Recht und Gesetz mit Füßen tritt. Eine ähnliche, aus unserer Sicht mittelalterliche Entwicklung in Bengalen, im Osten Indiens, ausgehend von der kommunistischen Partei CPI (M) wird hoffentlich im nächsten Jahrbuch präsentiert.

Zwei Arbeiten über die in der Verfassung verbotenen Kasten zeigen diese einerseits als vor allem Heirats- und Interaktionskreis in der Bauerngesellschaft mit abnehmender Tendenz (so Jürgensmeyer / Rösch) und andererseits als lebendige politische Gruppen, welche die Ausbildungsplätze, die Zusammensetzung des Gemeinderates, Zugang zu Krediten, Namensgebung öffentlicher Institutionen beeinflussen (Bronger mit Tabellen und viel Literatur). J. Malik beschreibt demgegenüber die soziale Lage der Muslime. Der sich auf den vor über 300 Jahren gegen den Mogulkaiser Aurangzeb revoltierenden Shivaji berufende militante Hinduismus bringe die vielfältigen Varianten zu einem fast monolithischen Islam zusammen. Es gehe nicht um Religion an sich, um das Sakrale, sondern um profane Interessen, die religiös artikuliert würden.

Zwei Beiträge beschäftigen sich mit dem eigentlichen Hauptproblem Indiens, der Bildung und Ausbildung: zunächst H.C. Rieger, Probleme des indischen Bildungswesens. Immer wieder wurden Kommissionen eingesetzt, deren Vorschläge oft auch realitätsnah und selbstkritischen waren, aber nach langen Beratungen schließlich in den Akten dahindämmerten. Auch von der gegenwärtigen Regierung gibt es einen diesbezüglichen Special Action Plan. Aber noch immer lebt in Indien die größte Zahl von Analphabeten weltweit mit großen Ungleichheiten zwischen den Geschlechtern, Stadt und Land und zwischen den einzelnen Bundesstaaten. Eine sehr negative Rolle spielt die hohe Quote der Schulabbrecher, weil die Kinder zum Familienunterhalt beitragen müssen oder weil (häufig) die Qualität des Schulunterrichts zu gering ist. Politik und Verwaltung haben 50 Jahre lang versagt, aber auch die Öffentlichkeit: Dies hat Nobelpreisträger Amartya Sen dieser Tage auch den Journalisten vorgeworfen.

S. Gosalia, Globalisierung und Braindrain im Kontext der Bildungspolitik in Indien, hebt zwei Aspekte hervor. Indische Eltern, die es sich leisten können, sorgen für die Ausbildung ihrer Kinder durch Förderung entsprechender Ausbildungseinrichtungen in den Städten und durch Studium im Ausland, besonders USA, Großbritannien, Kanada und Australien (Kosten angeblich $1 \mathrm{Mrd}$. DM/Jahr). Auch veranstalten inzwischen ausländische Universitäten und Stiftungen „Bildungsmessen“ in den indischen Metropolen, um Studenten anzu- 
locken. Der andere Aspekt ist die große finanzielle Stärkung, die im Ausland lebende Inder durch Investitionen in der Heimat dieser angedeihen lassen.

Der außenpolitische Teil des Jahrbuchs beginnt mit einer Übersicht von C. Wagner. H. Kreft schreibt über „Südasien als sicherheitspolitische Herausforderung“, dann C. Wagner über „Zwischen Konflikt, Kooperation und Realignment: US-Außenpolitik gegenüber der indischen Union“; C.D. Maaß mit „Indiens ehrgeiziges Nuklearkonzept“" und D. Reetz: „Optionen für Indien und Pakistan in Kashmir: Anatomie eines Konfliktes“. In diesen Beiträgen geht es in erster Linie um Kaschmir, die indischen Nuklearversuche, Indiens Verhältnis zu seinen Nachbarstaaten einschließlich China und um die beiderseits angestrebte Neugestaltung der Beziehung Indiens zur USA.

Zur Außenpolitik gehört auch die Darstellung H.-G. Wiecks über die Bemühungen, den Anrainern des Indischen Ozeans eine ähnliche Regionalbindung zu geben wie für Atlantik lang geschehen und für den Pazifik im Aufbau (APEC): die Indian Ocean Litoral Association of Regional Cooperation (IOLARC) mit zur Zeit 19 Mitgliedstaaten hat 1999 und 2000 Konferenzen abgehalten, und mehrere nicht am Indischen Ozean liegende Großmächte haben Assoziierung beantragt.

Für die binnen- und außenwirtschaftliche Entwicklung Indiens bietet E. Bierbrauer auf 15 Seiten eine konzentrierte Übersicht, einschließlich der Beziehung zu Deutschland. W.P. Zingel, Indien als Dienstleistungsexporteur, sieht Indiens Chancen im Export von Software-Arbeit und Computerfachleuten weiterhin steigen, ohne dass zu viele Hochqualifizierte das Land verlassen, denn vier Fünftel der Migranten kehrten zurück.

Angesichts der unerträglichen Luft- und Wasserverschmutzung besonders in den indischen Großstädten ist das von M. v. Hauff behandelte Thema „Relevanz erneuerbarer Energieträger" von enormer Wichtigkeit. Indien hat dank der geografischen Gegebenheiten ein großes Potential für Solar- und Windenergie. Wie in anderen Kohle produzierenden Ländern wird der Bergbau jedoch hoch subventioniert, und die neuen, ungleich besseren Energiequellen bleiben teuer.

Den Abschluss bildet eine historische und aktuelle Darstellung „Deutsch-indische Joint Ventures“ durch D. Matter, Geschäftsführer der Deutsch-Indischen Handelskammer in Düsseldorf. Im Anhang finden sich politische Chronik sowie Auswahlbibliographie und Statistiken für wirtschaftliche Fragen.

Die Lektüre des Bandes bringt wie seine beiden Vorgänger interessante Informationen und Darstellungen. Aber wieder drängt sich der Eindruck eiliger Zusammenstellung auf. Die Beiträge sind von (auch sprachlich) unterschiedlichem Niveau und nicht aufeinander abgestimmt. Man vermisst redaktionelle Bearbeitung. 


\section{Patrick Köllner (Hrsg.)}

\section{Korea 2000}

Politik, Wirtschaft, Gesellschaft

Institut für Asienkunde, Hamburg, 2000, 273 S., DM 48,--

Der Herausgeber selbst und zehn weitere sachkundige Autoren - darunter drei Koreaner haben auch diesmal wieder eine informative und gut zu lesende Bestandsaufnahme vorgelegt. Dass nur eines der 13 Kapitel sich mit Nordkorea beschäftigt, ist bedauerliche Folge der Selbstabschottung des dortigen Regimes. Welche Öffnungsimpulse dem Gipfeltreffen vom Juni 2000 folgen werden, bleibt abzuwarten.

Einen direkten Vergleich zwischen den beiden ungleichen Teilstaaten liefern die vorgeschaltete, vom Herausgeber zusammengestellte „Chronik der Ereignisse auf der koreanischen Halbinsel 1999“ und - besonders prägnant - der „Vergleich der Wirtschaft in Nordund Südkorea (1999)“ im Anhang: Wie hoffnungslos der Norden dem Süden hinterherhinkt, wird besonders deutlich am BIP pro Kopf (714 : 8581 US\$) und beim Außenhandelsvolumen (1.48: 263.44 Mrd. US\$), wobei schon kaum noch beachtenswert ist, dass Nordkorea einen Import-, Südkorea aber einen Exportüberschuss aufweist.

Von den fünf Kapiteln zur Innenpolitik Südkoreas bieten die einander gut ergänzenden vier Beiträge von Manfred Pohl, Hamburg (Südkoreas Innenpolitik 1999/2000: Schwerpunkte und Tendenzen), Huh Young, Seoul (Parteienstaatlich-repräsentative Demokratie und die Wahl-Idee und Wirklichkeit in der Republik Korea), Patrick Köllner, Hamburg (Informelle Politik in Südkorea: Allgemeine Anmerkungen und das Beispiel der Nationalversammlung) und Roland Wein, Essen (Die zivilgesellschaftlichen Organisationen in der Zeit der „Regierung des Volkes“ von Kim Dae-jung 1998-2000 - Eine Zwischenbilanz der Aktivitäten und Aufgaben) ein anschauliches Bild von der oft widersprüchlichen, insgesamt aber positiven Entwicklung Süd-Koreas zu einem nicht nur demokratisch verfassten, sondern auch demokratisch funktionierenden Gemeinwesen. Dagegen bietet Kim Man-su, Bochum (20. Jahrestag des Kwangju-Aufstandes: Achillesferse der südkoreanischen Demokratie oder ein Mythos?) eine sehr subjektive, aber in ihrer Ehrlichkeit überzeugende Bewertung der noch immer nicht aufgearbeiteten traumatischen Ereignisse vom Mai 1980.

Oliver Schramm, Seoul (Grundzüge und Tendenzen der südkoreanischen Außenpolitik) geht zunächst ausführlich auf die innerkoreanischen Entwicklungen ein und betont den Durchbruch des Gipfeltreffens vom 13.-15. Juni 2000. Bemerkenswert sei die betont zur Schau getragene Umgänglichkeit Kim Jong-ils gewesen, mit der er sich als internationaler Gesprächspartner habe empfehlen wollen. Anschließend werden die Beziehungen zu den USA, China, Japan und Russland sowie den EU-Staaten dargestellt und auf das internationale Engagement des Landes (Ost-Timor-Einsatz, ASEAN+3-Initiative) hingewiesen. Der Inhalt des Beitrags von Heinrich Kreft, Berlin (Die Beziehungen zwischen der EU und Südkorea: Enge wirtschaftliche und beginnende politische Kooperation) wird durch den Titel zutreffend umschrieben. 
Von den fünf der Wirtschaft gewidmeten Beiträgen befassen sich drei mit allgemeinen Problemen: Patrick Köllner, Hamburg bietet in sehr einprägsamer Form „,Schlaglichter der Wirtschaft Südkoreas 1999/2000“, Peter Tergeist, OECD Paris (Industrielle Beziehungen und Arbeitsrechtsreform in Korea) erkennt in der Entwicklung der Beziehungen zwischen Unternehmern und Gewerkschaften die Tendenz, von der bedingungslosen Konfrontation zu mehr Zusammenarbeit zu kommen, und Peter Mayer / FES Seoul (Lehren aus der Währungs- und Finanzkrise - Südkorea und die Reform der internationalen Finanzarchitektur) konstatiert eine vorsichtige Öffnung des zuvor völlig intransparenten Finanzsektors und dessen dadurch ermöglichte Eingliederung in das internationale Finanzsystem.

Park Byeung-Kwan / Seoul (Bewertung deutscher Direktinvestitionen während der koreanischen Wirtschaftskrise 1998 - Eine finanzwirtschaftliche Analyse) und Tim Goydke, Duisburg (Die Auswirkungen der Koreakrise auf die Geschäftstätigkeit deutscher Unternehmen - Eine empirische Untersuchung) zeichnen mit vielen einprägsamen Schaubildern ein Panorama der gerade im Krisenjahr 1998 dramatisch angewachsenen Tätigkeit deutscher Unternehmen in Korea und kommen dabei zu überwiegend positiven Prognosen. Auf nur zehn Seiten behandelt Manfred Pohl, Hamburg „Die Wirtschaft Nordkoreas 1999/2000“ und bestätigt deren nach wie vor katastrophalen Zustand: Zwar war 1999 erstmals seit zehn Jahren ein leichter Anstieg des BSP zu verzeichnen, doch lag die Industrieproduktion nur bei $75 \%$ der von 1989! Konkrete Auswirkungen der auf dem innerkoreanischen Gipfel vom Juni 2000 proklamierten Öffnung sind noch nicht feststellbar. Man darf gespannt sein, ob Lichtblicke wie die von George Soros gesponserte Ausbildung nordkoreanischer Wirtschaftsfachleute in Ungarn zu einer Aufhellung des ganzen Horizonts führen werden. Insgesamt hat dieser Band das in den Vorjahren etablierte hohe Niveau gehalten. Dass der Herausgeber bestrebt ist, dies auch künftig zu tun, dokumentiert sein am Schluss abgedrucktes Call for papers.

Karl Leuteritz, Königswinter

Ostasiatischer Verein e.V. (OAV) (Hrsg.)

Wirtschaftshandbuch Asien Pazifik 2000/2001

45. Ausgabe, Hamburg, 2000, 702 S., DM 129,--

Zum 45. Mal legt der Ostasiatische Verein (OAV) sein Asien-Pazifik-Handbuch vor, dessen 26 Länderkapitel alphabetisch von Australien bis Vietnam und geographisch von Indien bis zu den südpazifischen Inselländern reichen. Trotz gestiegenen Umfangs ist der Band durchaus noch handlich geblieben, was den Zugang zu dem hochinteressanten Informationsmaterial sehr erleichtert.

Vorgeschaltet sind zwei allgemein orientierende Einleitungskapitel, die man vor dem Zugriff auf die jeweils interessierenden Länderdaten nicht überschlagen sollte: „Ostasien 
2000: Lehren am Ende einer Krise“ (Rüdiger Machetzki) und „Die ASEAN auf dem Weg zur Erneuerung“ (Klaus-A. Pretzell). Wichtig ist auch der umfangreiche Adressenteil (S. 629-702). In dessen Auflistung der diplomatischen (und konsularischen!) Vertretungen erscheint leider Bhutan - im Gegensatz zu anderen Ländern ohne Vertretungen - überhaupt nicht, und bei der Republik Korea ist die Außenstelle Bonn der Botschaft Berlin nicht berücksichtigt.

Dann werden in den 26 Länderkapiteln 37 Länder und Territorien behandelt: Einerseits müssen sich die 16 südpazifischen Inselländer mit einem gemeinsamen Kapitel begnügen, andererseits sind neben der VR China auch den kürzlich eingegliederten Sonderzonen Hongkong und Macao je ein eigenes Kapitel gewidmet. hierfür könnten 16 ausgewiesene Fachleute als Verfasser gewonnen werden. Die Kapitel folgen - wie in den Vorjahren einer einheitlichen Gliederung:

Am Anfang stehen eine Zusammenstellung der „Grunddaten“, Stichworte zur aktuellen „wirtschaftlichen und politischen Lage“ und ein „Statistisches Profil“. Der folgende Textteil unterschiedlicher Länge (z.B. 17 Seiten für Japan, 7 Seiten für Bhutan; je 14 Seiten für Nord- und Südkorea) bietet zunächst einen politischen Überblick und behandelt dann Wirtschafts- und Finanzpolitik, die jeweils wichtigsten Wirtschaftszweige, die Außenwirtschaft und die Beziehungen zu Deutschland. Dem folgt eine Darstellung der Marktzugangsbedingungen. Statistische Tabellen über den bilateralen Außenhandel und den Gesamtaußenhandel nach Waren und nach Ländern schließen die Darstellung ab.

In der „Demokratischen Volksrepublik Korea“ (Nordkorea) stellt Manfred Pohl (S. 269281) den Beginn einer vorsichtigen Öffnung des bisher völlig abgeschlossenen Systems fest - erstmals wurde 1999 ein Haushaltsdefizit eingeräumt! -, warnt aber vor euphorischen Erwartungen nach dem Nord-Süd-Gipfeltreffen vom Juni 2000. Erstmals seit 1989 zeigte das BIP 1999 ein reales Wachstum (6,2 \%), betrug mit 130 Mrd. US\$ aber nur wenig mehr als die Hälfte der vor zehn Jahren erzielten Summe.

Von fast noch größerer Skepsis zeugen die Ausführungen des OAV-Repräsentanten in Pyongyang, Günter Unterbeck, über die Marktzugangsbedingungen. Demgegenüber zeigte die Bestandsaufnahme für die „Republik Korea“ (Südkorea) von Patrick Köllner trotz berechtigter Hinweise auf fortbestehende Risiken ein durchgehend positives Bild: Aus der schwersten Rezession seit dem Koreakrieg kommend, schaffte das Land 1999 mit 10,7 \% das weltweit höchste Wirtschaftswachstum!

Auch für die Marktzugangsbedingungen stellt Florian Schuffner, langjähriger Geschäftsführer der Deutsch-Koreanischen IHK Seoul, eine stetige Verbesserung fest. Bei auftretenden Schwierigkeiten dürfte sich das neu geschaffene Amt des Office of the Investment Ombudsman als nützlich erweisen.

Auch die neue Ausgabe des Wirtschaftshandbuchs ist für deutsche Unternehmen mit AsienInteresse ein unverzichtbares Werkzeug.

Karl Leuteritz, Königswinter 